\title{
Hypersonic Vehicle Flight Dynamics with Coupled Aerodynamics and Reduced-order Propulsive Models
}

\author{
Derek J. Dalle, Scott G. V. Frendreis $\dagger$ James F. Driscoll Carlos E. S. Cesnik $^{\S}$ \\ University of Michigan, Ann Arbor, MI 48109
}

\begin{abstract}
A new model is developed to more accurately capture the dynamics and control of an air-breathing hypersonic vehicle using a computationally inexpensive formulation. The vehicle model integrates a scramjet engine analysis tool developed specifically for use in a control-oriented model and a six-degree-of-freedom rigid-body flight dynamics model. The combined hypersonic vehicle model requires less than ten seconds with a single $2.6 \mathrm{GHz}$ processor to calculate the total thrust, lift, and aerodynamic moment on the vehicle. The inlet and nozzle analysis handles shock-shock and shock-expansion interactions, and expansions are considered to be a series of discrete waves. The combustor model utilizes scaling laws that retain some of the fidelity of higher-order simulations. On the parts of the vehicle that are not part of the propulsive flowpath, modified shock-expansion theory is used to calculate the pressure. In this approach the role of the propulsive model will be only to calculate the net forces and moments on the inlet, combustor, and nozzle. The result is a control-oriented hypersonic vehicle model that qualitatively captures the nonlinear interactions between vehicle dynamics and the scramjet engine.
\end{abstract}

\section{Nomenclature}
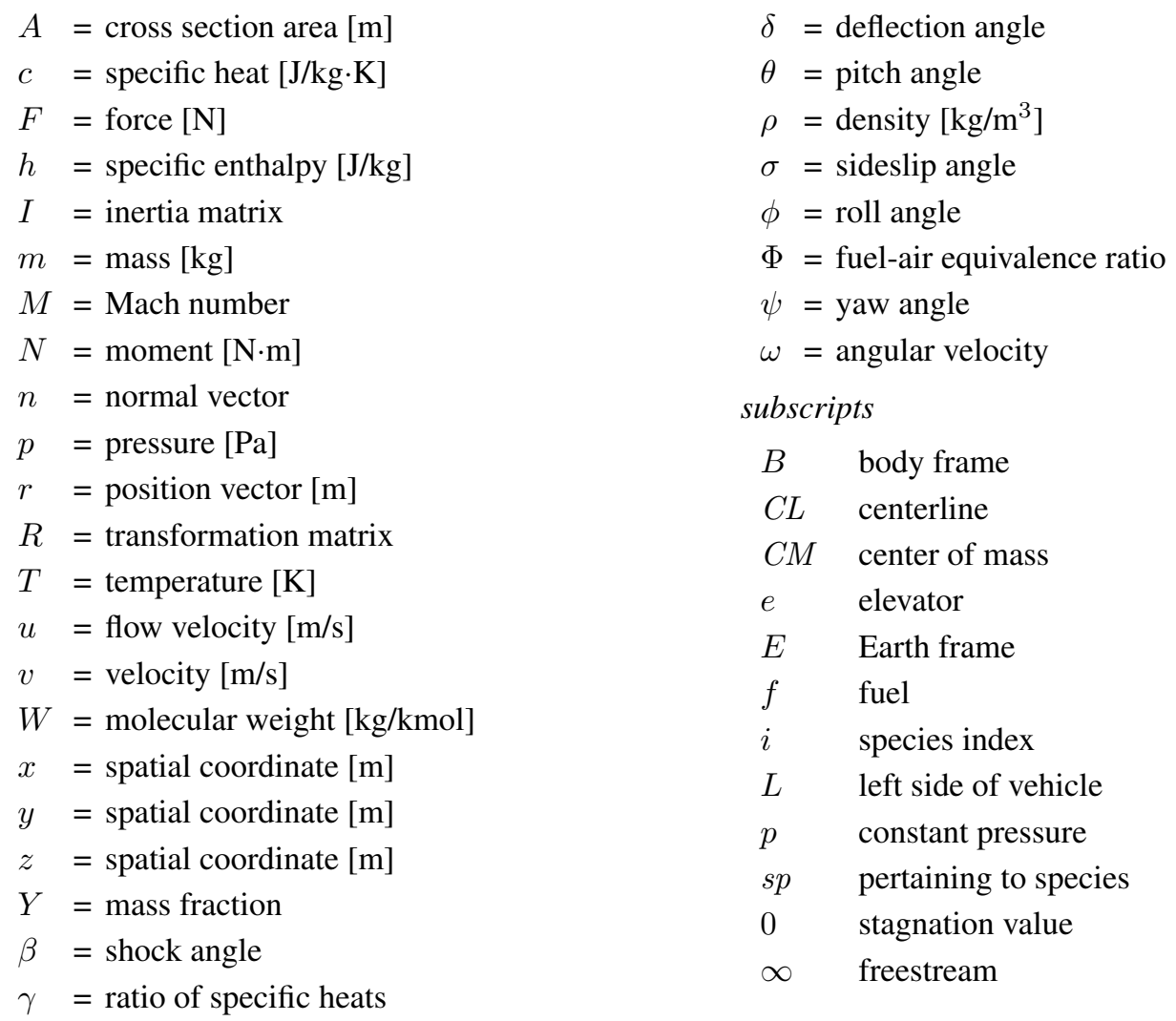

$B$ body frame

$C L$ centerline

$C M$ center of mass

e elevator

E Earth frame

$f$ fuel

$i \quad$ species index

$L \quad$ left side of vehicle

$p \quad$ constant pressure

$s p \quad$ pertaining to species

0 stagnation value

$\infty \quad$ freestream

*Graduate Research Assistant, Department of Aerospace Engineering, Member AIAA, dalle@umich . edu

${ }^{\dagger}$ Graduate Research Assistant, Department of Aerospace Engineering, Member AIAA, scottgvf@umich.edu

${ }^{\ddagger}$ Professor, Department of Aerospace Engineering, AIAA Fellow, james fd@umich . edu

$\S$ Professor, Department of Aerospace Engineering, AIAA Associate Fellow, cesnik@umich. edu 
overscripts

$(\vec{\cdot}) \quad$ vector quantity

$(\hat{.)}$ unit vector

$(\cdot)$ skew-symmetric matrix

$(\cdot)$ time derivative superscripts

$(\cdot)^{\prime} \quad$ quantity per unit width

\section{Introduction}

Among the many challenges facing the development of air-breathing hypersonic vehicles is maintaining controlled flight. In addition to maximizing fuel efficiency, ensuring adequate thrust and lift, and limiting heat transfer, the vehicle has to be able to balance all of the forces on it. In order to balance these forces in a simulation, a model is required that can evaluate the performance of the entire vehicle in a few seconds or less.

Additionally, flight at such high speeds causes complex interactions among the airframe, propulsions system, vehicle aerodynamics, and other aspects. As a result, it is not possible to design each component of the vehicle independently. Therefore a fast model that can analyze a range of vehicles can also be useful to assist the design of a hypersonic vehicle. The overall goal of this hypersonic vehicle modeling effort is to create a three-dimensional flight simulation framework that includes coupled inertial, aerodynamic, propulsive, elastic, and thermal analysis in such a way to enable accurate control simulation. The model should be accurate to within $10 \%$ and able to analyze a vehicle in a few seconds or less on a single-processor computer.

In addition to high-fidelity hypersonic vehicle analysis by Candler et al., ${ }^{1,2}$ Higgins and Schmidt, ${ }^{3}$ and many others, there has been a considerable effort to develop low-order hypersonic vehicle simulations that can run in short time periods using only moderate computational resources. Oppenheimer et al. ${ }^{4,5}$ and Chavez and Schmidt ${ }^{6}$ have developed longitudinal (two-dimensional) hypersonic flight dynamics models that can be used to simulate a variety of vehicle trajectories. Mirmirani et al. $^{7}$ investigated merging experimental and computational data to generate control algorithms for hypersonic vehicles.

At the same time, progress has been made by O'Brien et al. ${ }^{8}$ and Torrez et al. ${ }^{9,10}$ in developing fast simulations of air-breathing hypersonic propulsion systems. The present work aims to extend a three-dimensional flight dynamics mode $^{11}$ by including a more accurate propulsion model. Our approach is to separate the vehicle into portions that are part of the propulsive system and portions that are not. We model the propulsive components, i.e., the inlet, ${ }^{12}$ combustor, ${ }^{10}$ and nozzle, ${ }^{13}$ using a two-dimensional representation that has the required fidelity for propulsive analysis. We analyze the remaining portions of the vehicle using a simpler three-dimensional model that only calculates the pressure and temperature on the vehicle surfaces.

The use of a two-dimensional model for some parts of the vehicle limits the types of geometries that the proposed model can analyze. For example, the approach cannot be used for a vehicle with an inward-turning inlet ${ }^{2}$ or a conicaltype nozzle. However, for hypersonic vehicles similar to those shown in Fig. 1, the proposed approach should be relatively accurate because the portions that are modeled as two-dimensional do not vary much in the one of the directions normal to the freestream flow. In this paper all analysis is of the geometry provided by VSI Aerospace, Inc. ${ }^{14}$ which is shown in Fig. 1.

\section{Hypersonic Vehicle Model}

The focus of this paper is to integrate a three-dimensional, six-degree-of-freedom flight dynamics model, an unsteady aerodynamic model, and a control-oriented propulsion model. The flight dynamics model consists of the six-degree-of-freedom rigid body equations of motion coupled with a simple unsteady aerodynamic model. The aerodynamic model determines the aerodynamic forces and moments acting over the vehicle. However, because the inlet, isolator, combustor, and nozzle are so tightly coupled, the propulsion model is responsible for analyzing the inlet and nozzle in addition to the isolator and combustor.

In our proposed model, the vehicle itself consists of many polygonal surfaces. Each of these polygons is either a part of the propulsive system, part of the remaining vehicle, or on the border between the two. The results of both components are combined to generate a net force and moment on the vehicle, which can be used in a rigid-body flight dynamics simulation. This is an improvement over previous formulations because it combines a fast, higher-fidelity model of the propulsion system with a model of a complete hypersonic vehicle in a six-degree-of-freedom simulation framework. 


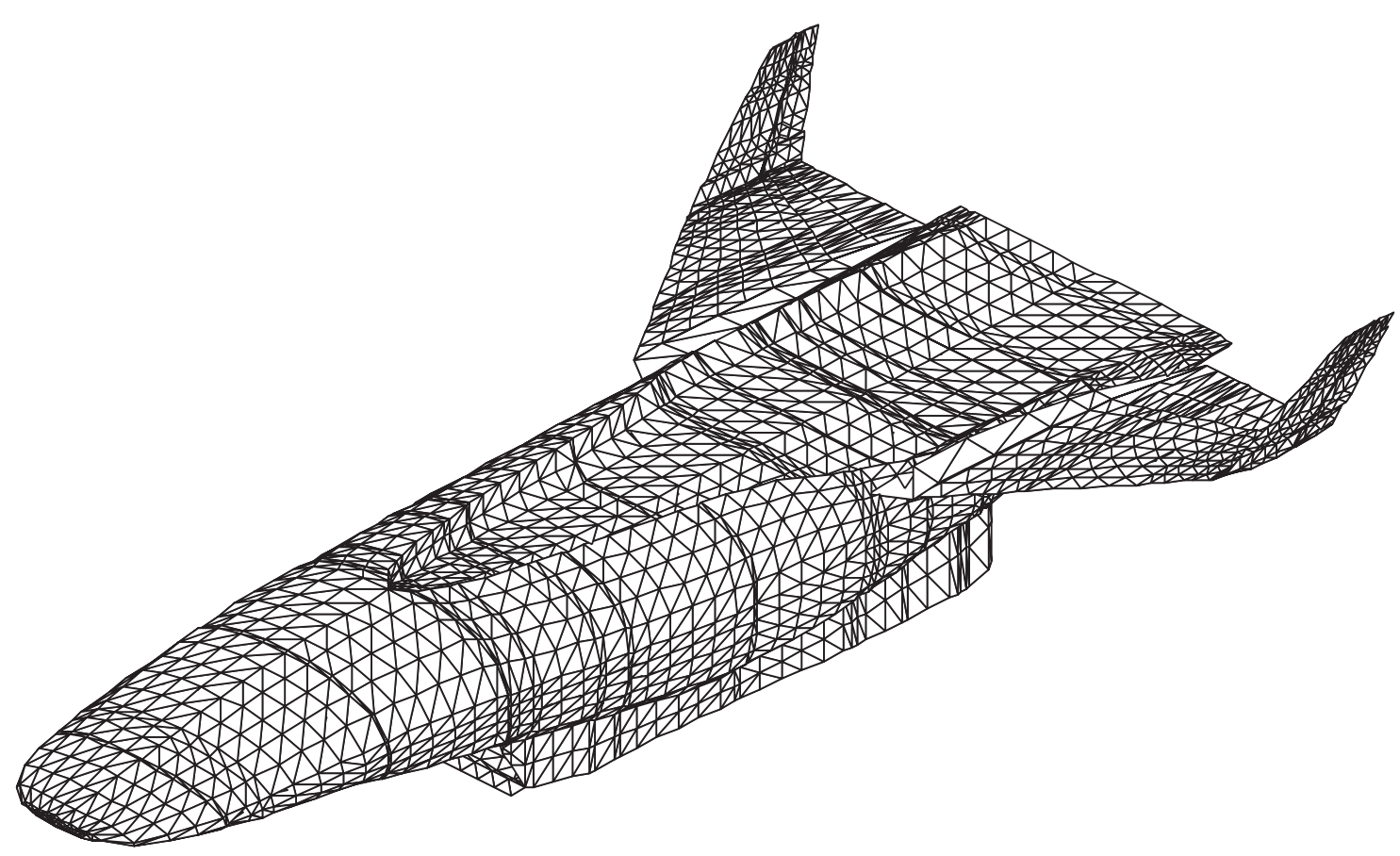

Figure 1. Isometric view of hypersonic vehicle used in this analysis.

\section{A. Rigid Body Equations of Motion}

The six-degree-of-freedom rigid body equations of motion are derived using a Newtonian approach, assuming a flatearth inertial reference frame. The translational velocity vector, $\vec{v}_{B}$, and rotational velocity vector, $\vec{\omega}_{B}$, are expressed in a body-fixed reference frame whose origin is located at an arbitrary fixed point on the vehicle. During long-duration simulation of free flight, a hypersonic vehicle is expected to consume a significant portion of its total mass in fuel. Therefore the rigid body equations of motion are written for a vehicle with varying inertial properties (total mass, center of mass location, etc.). These equations of motion take the form

$$
\left[\begin{array}{cc}
m & -m \tilde{r}_{C M} \\
m \tilde{r}_{C M} & I_{B}
\end{array}\right]\left\{\begin{array}{l}
\dot{\vec{v}}_{B} \\
\dot{\vec{\omega}}_{B}
\end{array}\right\}+\left[\begin{array}{cc}
m \tilde{\omega}_{B} & -m \tilde{\omega}_{B} \tilde{r}_{C M} \\
m \tilde{r}_{C M} \tilde{\omega}_{B} & \tilde{\omega}_{B} I_{B}
\end{array}\right]\left\{\begin{array}{l}
\vec{v}_{B} \\
\vec{\omega}_{B}
\end{array}\right\}=\left\{\begin{array}{l}
\vec{F}_{B} \\
\vec{N}_{B}
\end{array}\right\}
$$

where $m$ is the vehicle mass, $r_{C M}$ is the position of the center of mass with respect to the body-fixed reference frame, $(\tilde{\cdot})$ is the skew-symmetric matrix operator, and $I_{B}$ is the inertia matrix with respect to the center of mass. The external forces, $\vec{F}_{B}$, and moments, $\vec{M}_{B}$, come the vehicle aerodynamics, propulsion system, and gravity.

The aircraft attitude is expressed using the roll $(\phi)$, pitch $(\theta)$, and yaw $(\psi)$ Euler angles. The Euler angle kinematics are related to the rigid body rotational velocity by

$$
\left\{\begin{array}{c}
\dot{\phi} \\
\dot{\theta} \\
\dot{\psi}
\end{array}\right\}=\left[\begin{array}{ccc}
1 & \sin (\phi) \tan (\theta) & \cos (\phi) \tan (\theta) \\
0 & \cos (\phi) & -\sin (\phi) \\
0 & \frac{\sin (\phi)}{\cos (\theta)} & \frac{\cos (\phi)}{\cos (\theta)}
\end{array}\right]\left\{\begin{array}{l}
\omega_{B, x} \\
\omega_{B, y} \\
\omega_{B, z}
\end{array}\right\}
$$

The position of the vehicle is expressed in terms of the earth-fixed inertial reference frame. The inertial position kinematics are related to the Euler angles and rigid body translational velocity through

$$
\left\{\begin{array}{l}
\dot{x}_{E} \\
\dot{y}_{E} \\
\dot{z}_{E}
\end{array}\right\}=R_{E B}(\phi, \theta, \psi)\left\{\begin{array}{l}
v_{B, x} \\
v_{B, y} \\
v_{B, z}
\end{array}\right\}
$$

where $R_{E B}$ is the body-to-Earth transformation matrix. 


\section{B. Unsteady Aerodynamic Model}

We have developed a simple modification to two-dimensional shock-expansion theory to develop a model that is relatively accurate in all regions and based on well-established compressible flow physics with minor modifications. This method is a local inclination method, i.e., the pressure at any point on the surface of the vehicle is determined by that point's total velocity and orientation (and independent of the geometry of the rest of the vehicle). For each surface, as shown in Fig. 1, a local pressure is calculated, which is multiplied by the area of the triangle to determine the force applied on each surface. Then the incident velocity on the surface is

$$
\vec{v}=\vec{v}_{\infty}+\vec{\omega} \times \vec{r}
$$

where $\vec{v}_{\infty}$ is the freestream (vehicle) velocity, $\vec{\omega}$ is the angular velocity of the vehicle, and $\vec{r}$ is the vector from the vehicle's center of mass to the center of the current surface. The deflection angle $\delta$ is given by

$$
\sin \delta=-\frac{\hat{n} \cdot \vec{v}}{\|v\|}
$$

where $\hat{n}$ is the unit normal to the surface. We then convert the incident velocity into a Mach number using

$$
M=\|v\| \sqrt{\frac{\rho_{\infty}}{\gamma p_{\infty}}}
$$

where $\gamma$ is the specific heat ratio. If $\delta<0$, Prandtl-Meyer theory is used. Otherwise the pressure is calculated using the formula

$$
p=p_{\infty}\left(\frac{2 \gamma}{\gamma+1} M^{2} \sin ^{2} \beta-\frac{\gamma-1}{\gamma+1}\right)
$$

where $\beta$ is a shock angle. If the deflection angle is sufficiently small, it is possible for an attached shock wave to exist, and the shock angle calculated using the implicit formula

$$
\tan \delta=2 \cot \beta \frac{M^{2} \sin ^{2} \beta-1}{M^{2}(\gamma+\cos 2 \beta)+2}
$$

The maximum value for the shock angle is

$$
\sin ^{2} \beta_{\max }=\frac{(\gamma+1) M^{2}-4+\sqrt{(\gamma+1)^{2} M^{4}+8\left(\gamma^{2}-1\right) M^{2}+16}}{4 M^{2} \gamma}
$$

which leads to a maximum deflection angle by substituting the result of (9) into (8). If the deflection angle is too large for an attached shock, i.e., $\delta>\delta_{\max }$, the wave angle is given by an interpolation;

$$
\beta=\beta_{\max }+\frac{\delta-\delta_{\max }}{\pi / 2-\delta_{\max }}\left(\pi / 2-\beta_{\max }\right)
$$

This aerodynamic model corresponds to a normal shock if the deflection angle is exactly $90^{\circ}$. In light of this, our model avoids large errors for all possible inclination angles.

\section{Propulsion Model}

The propulsion model can further be split into three components: the inlet, the combustor, and the nozzle. In the scramjet mode, neither component affects the one upstream of it, and the three parts can be solved in sequence. In ramjet mode the flow through the combustor section is subsonic, and signals can travel upstream, but we are only considering scramjet-mode operation in the present analysis. This also means that the isolator, which is the portion of the engine flowpath between the inlet and the combustor section, can be largely ignored. The station numbering associated with the propulsion model is shown in Fig. 2. 


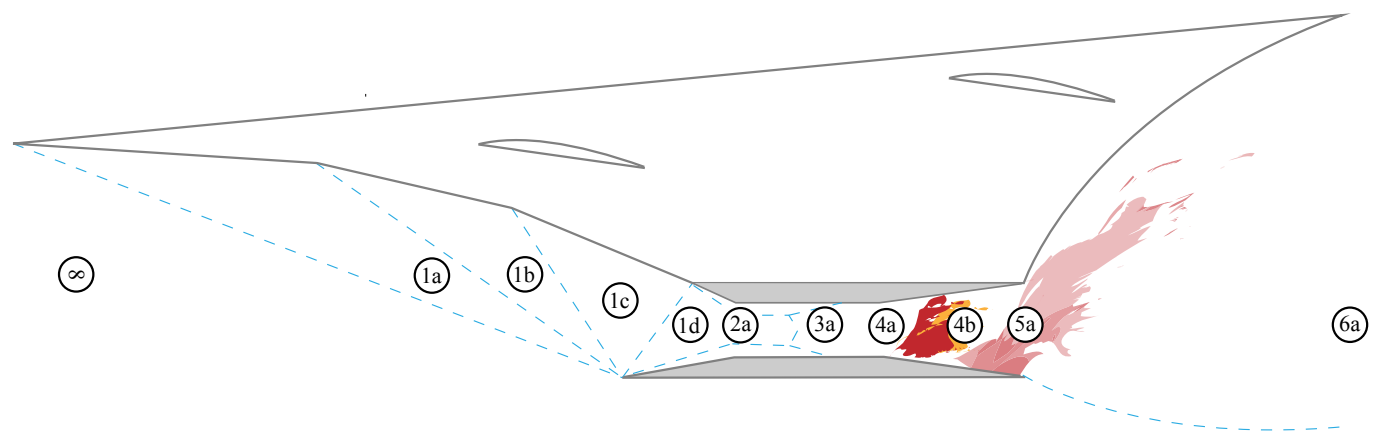

Figure 2. An example geometry. The station numbers are extensible by adding successive letters to each station for a more complex geometry.

\section{Inlet}

The inlet model considers scramjet inlet designs that are approximately two-dimensional. A satisfactory controloriented model must require a relatively small amount of computational time and still yield a relatively accurate solution for the inlet flow. Instead of solving directly for the flow conditions at each point in the flow, as is done in computational fluid dynamics (CFD), our method solves for the positions of the relevant waves, which separate regions in which the flow properties are considered to be uniform. The locations of the shock waves and expansions are determined using established two-dimensional supersonic theory.. To make this possible in a digital computing environment, expansion fans are approximated as a number of discrete isentropic waves. In many ways this is a generalized and automated version of the method of characteristics. This method has been described in several previous papers. ${ }^{10,13,15,16}$

The output of this inlet model is a set of polygons, each with an associated set of thermodynamic conditions (density, pressure, temperature, Mach number, and flow direction). The result resembles the output of two-dimensional CFD, as shown in the comparison in Fig. 3. The reduced-order solution and CFD solutions are shown for the same inlet flying at a flight Mach number of $M_{\infty}=10.0$ and angle of attack of $\alpha=0$.

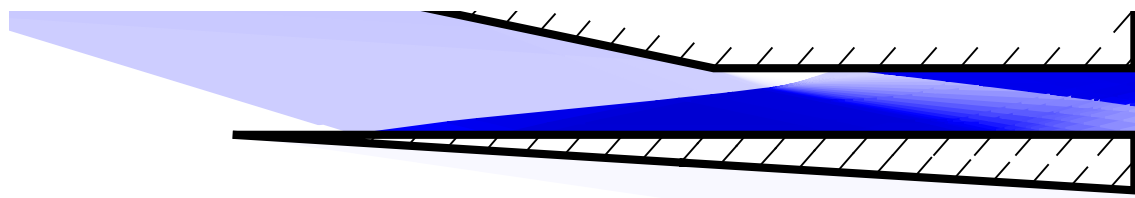

a) Proposed model solution

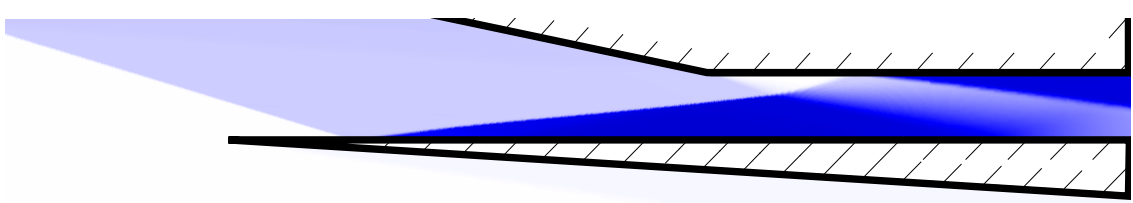

b) CFD Solution

Figure 3. Comparison between solution from proposed inlet model and high-fidelity CFD solution. Darker shades of blue represent regions of higher pressure; white represents freestream pressure and black represents $p / p_{\infty}=90$.

Of critical importance to the inlet model is the discretization of expansion fans. An expansion fan can be seen in both graphics of Fig. 3 starting at the corner in the upper surface. Prandtl-Meyer theory can be used to obtain the exact solution to this type of flow. However, this result is continuous, and needs to be modeled as a discrete change in some way to be useful in our model. Unfortunately, any attempt to discretize the Prandtl-Meyer expansion results in a approximation that does not conserve momentum. ${ }^{13}$ Our approach is to model the expansion as a series of weak expansion shocks, which is a close approximation to Prandtl-Meyer theory and conserves mass, momentum, and energy. The geometry and an example of a discretized expansion are shown in Fig. 4.

Another critical modeling problem occurs when two waves intersect each other. The inlet model resolves this 
situation by solving a two-dimensional Riemann problem. ${ }^{10}$ Finally, the combustor model assumes that the flow leaving from the inlet is uniform (i.e., does not vary with coordinates normal to the flow direction). To match this constraint with the two-dimensional result from the inlet analysis, we select the thermodynamic state in the combustor so that the total fluxes of mass, momentum, and energy are equal at the end of the inlet and the beginning of the combustor.

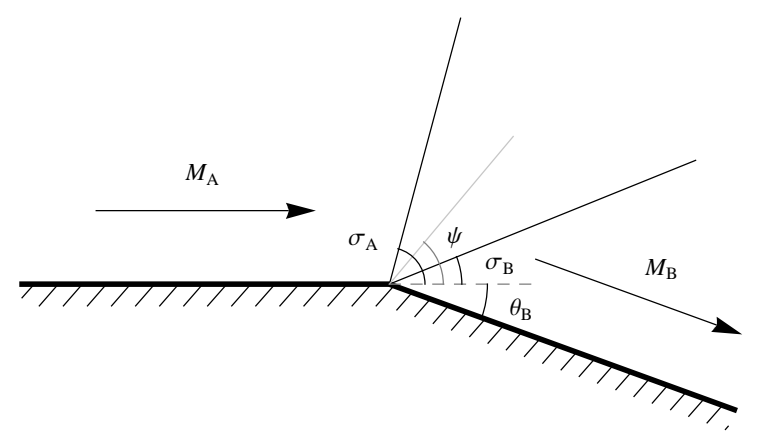

a) Geometry and definitions

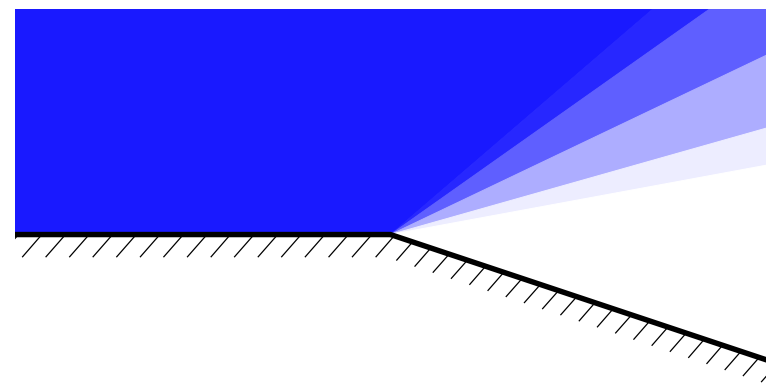

b) Example

Figure 4. Illustration of Prandtl-Meyer expansion wave. The geometry and nomenclature of the problem are shown 4a) while 4b) shows an example solution from the proposed inlet model in which the expansion fan is modeled as five discrete waves. Darker shades of blue represent higher pressures.

\section{Duct Solution}

The combustion code marches the flow conservation equations from the beginning of the combustor (station $3 \mathrm{a}$ in Fig. 2) to the end of the internal nozzle (station 5a). All flow states are allowed to vary in the downstream axial direction. To keep the model one-dimensional, only derivatives with respect to the axial coordinate are considered. Some quantities, such as jet spreading and mixing, vary in the transverse directions, but they may only vary algebraically such that their evolutions do not depend on the information propagating downstream.

The conservation equations, along with the equation of state, form the basis of the model. By taking derivatives in the $x$-direction, these give a set of ordinary differential equations. The simultaneous solution provides all the information needed to calculate the evolutions of the state variables $(p, \rho, T)$ and the heat release through the duct.

The two simplest differential equations used in the propulsion model follow. In these equations, $W$ is the average molecular weight of the gas, and $A$ is the area of the combustor duct as a function of $x$. The equation of state takes the form

$$
\frac{1}{p} \frac{d p}{d x}=\frac{1}{T} \frac{d T}{d x}+\frac{1}{\rho} \frac{d \rho}{d x}-\frac{1}{W} \frac{d W}{d x}
$$

which comes from differentiating the equation of state, $p=\rho R T$. The conservation of mass gives

$$
\frac{1}{\rho} \frac{d \rho}{d x}=\frac{1}{\dot{m}} \frac{d \dot{m}}{d x}-\frac{1}{u} \frac{d u}{d x}-\frac{1}{A} \frac{d A}{d x}
$$

where $\dot{m}$ is the mass flow rate through the duct, and $d \dot{m} / d x$ represents the mass flow rate of fuel added as a function of $x$. The total mass flow rate of fuel added is

$$
\dot{m}_{f}=\int \frac{d \dot{m}}{d x} d x
$$

The fuel-air equivalence ratio, $\Phi$, is the ratio of the fuel mass flow rate to the maximum amount of fuel that could burn based on the amount of oxygen in the combustor. The conservation of momentum states

$$
\frac{1}{u} \frac{d u}{d x}=-\frac{1}{\rho u^{2}} \frac{d p}{d x}-\frac{1}{\dot{m}} \frac{d \dot{m}}{d x}
$$

If we define a mass-averaged specific heat as

$$
\bar{c}_{p}=\sum_{i=1}^{n_{s p}} c_{p, i} Y_{i}
$$


and neglect viscous forces, the conservation of energy takes the form

$$
\frac{\bar{c}_{p}}{h_{0}} \frac{d T}{d x}=\sum_{i=1}^{n_{s p}}\left(\frac{1}{\dot{m}} \frac{d \dot{m}_{i}}{d x}-\frac{d Y_{i}}{d x}\right) \frac{h_{i}}{h_{0}}-\frac{u}{h_{0}} \frac{d u}{d x}-\frac{1}{\dot{m}} \frac{d \dot{m}}{d x}
$$

These are essentially the equations used in the combustor model. ${ }^{10}$ The rates of change for each species, $d Y_{i} / d x$, are calculated at each point in the combustor using a flamelet model.

\section{Nozzle}

To accurately predict thrust, a nozzle model must analyze the expansion and acceleration of the exhaust gases from the combustor. In a typical scramjet nozzle, the lower edge of the exhaust plume is a slip stream, and its location varies according to combustor and flight conditions. An example of a scramjet nozzle flow is shown in Fig. 5.

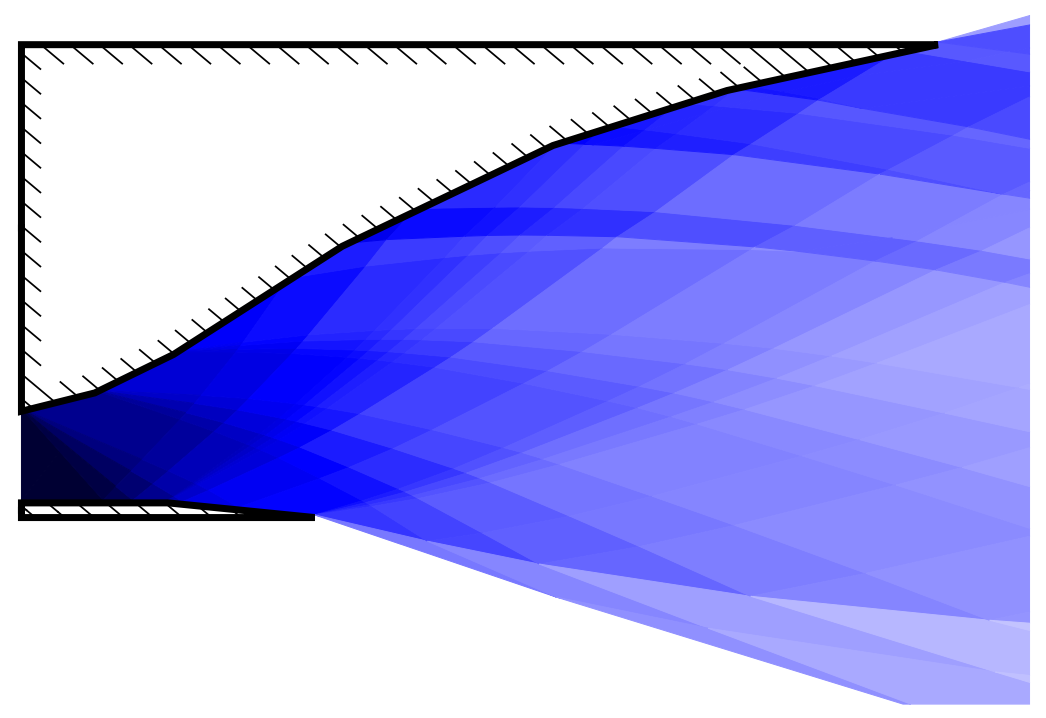

Figure 5. Reduced-order solution of example scramjet nozzle. Darker shades of blue represent higher temperatures.

The temperature in the combustor section reaches temperatures high enough to cause significant dissociation of the gas, which means that at the beginning of the nozzle, some of the combustion products will be split into smaller molecules. However, the temperature drops dramatically in the nozzle, and these smaller molecules, e.g., $\mathrm{N}$ and $\mathrm{O}$, will recombine into stable molecules such as $\mathrm{N}_{2}$ and $\mathrm{O}_{2}$. In most flows, the release of energy caused by such a chemical change would have a significant effect. However, the velocities are so large in scramjet nozzles that the chemical energy release is small compared to the kinetic energy of the flow. ${ }^{13}$ For that reason, and because it would slow down the computation, we do not consider chemistry in the nozzle.

In addition, it is crucial to determine the streamline that forms the lower edge of the exhaust plume. This streamline determines the effective area of the nozzle and thus is has a significant effect on the total thrust of the nozzle. Calculating this streamline using a one-dimensional model would be impossible because information in a supersonic flow travels along characteristics, which are the lines shown in Fig. 4.

Previous models have considered one-dimensional models with several simplifying assumptions. ${ }^{5,6,16}$ Because the nozzle does not have the sensitive behavior of the inlet, these one-dimensional models can produce a representative approximation of the forces on the nozzle. However, even if it were possible to determine the location of the exhaust plume, a one-dimensional model gives inaccurate thrust predictions. ${ }^{13}$ We solve this problem by modeling the nozzle using a slightly modified version of the inlet model, which accurately considers the two-dimensional nature of the nozzle. 


\section{Integration}

There are two largely disparate models in our proposed method of analysis for the hypersonic vehicle. In particular the models used in conjunction with the propulsion model are two-dimensional, and the models used with the aerodynamic model are three-dimensional. Since the aerodynamic model does not accurately compute the conditions in the gas away from the surface of the vehicle, it cannot be used to accurately model the propulsion. Likewise, the propulsion model cannot predict the three-dimensional forces necessary for a six-degree-of-freedom simulation.

Thus we are forced to use both models, and we must determine for each of the vehicle's surfaces which model to use. In addition, we must extract the two-dimensional geometries to use with the propulsion model. We have developed an automated utility to complete both of these tasks, and the result is visualized in Fig. 6. The surfaces in red are part of the inlet or nozzle, and the boundary between these regions and the rest of the vehicle is shown with a thick black line. The traces of the two-dimensional geometries used in the propulsive model are shown with dotted blue lines.

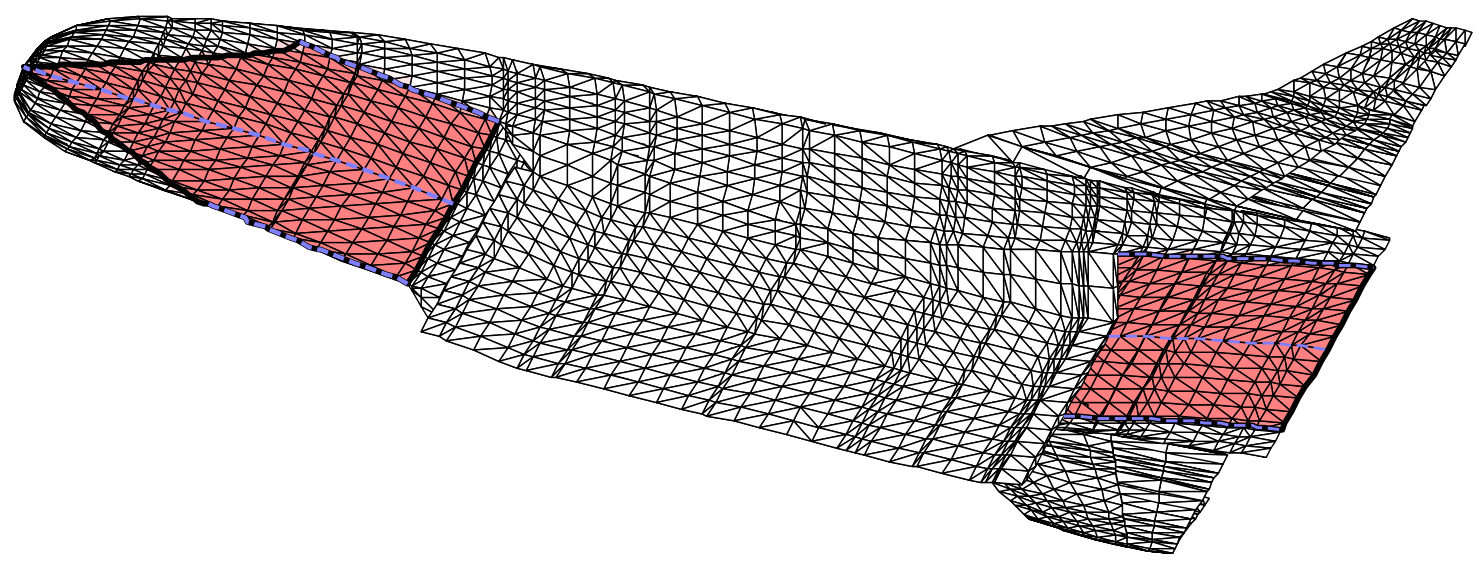

Figure 6. Lower side of hypersonic vehicle with cells in the propulsive flowpath shown as red and the two-dimensional cuts shown with the blue dashed line.

\section{A. Region Determination}

Determining which surfaces are part of the isolator and combustor is relatively easy because they are the surfaces surrounded by the cowl. Most of the work is in determining the geometry of the inlet and nozzle. Our general approach to this is to start with points on the edge of the inlet cowl or nozzle cowl and trace streamlines in the appropriate directions.

For instance, to trace the boundary of the inlet, we begin with the three points: the left, center, and right points of the inlet cowl. In Fig. 6, these are the two right-most corners of the inlet pentagon and the point in the middle of the edge joining those two corners. Starting with those three points, we trace the intersection of the vehicle and a plane containing that point and unit vectors pointing in the flow direction and vertical direction. This same process is repeated for the other two inlet cowl points, which generates the three blue dotted lines in the inlet region of Fig. 6. Finally, lines are traced along the surface to join the endpoints of the three blue dotted lines. Then the entire process is repeated for the nozzle.

\section{B. Engine Flowpath}

To limit the adverse effects of using a two-dimensional model on some parts of the vehicle, the propulsion code is run twice for each simulation of the vehicle. This is the purpose of the three dotted lines in Fig. 6. Note that only two calculations are necessary because the left and right flowpaths are identical to each other. The engine flowpath geometries are shown in Fig. 7. 


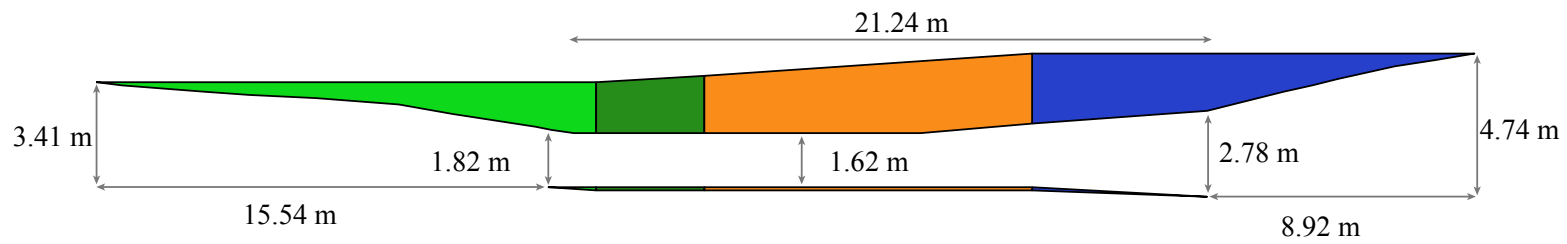

a) Centerline propulsive geometry

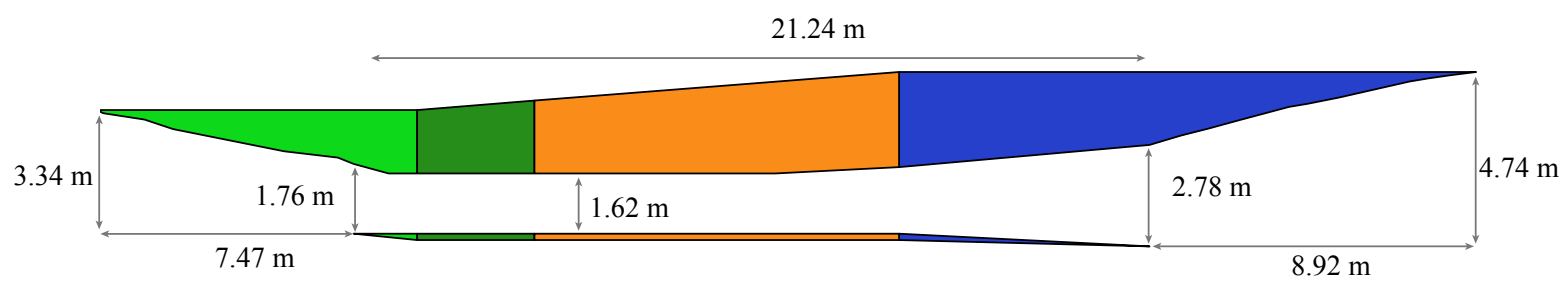

b) Left/right propulsive geometry

Figure 7. Schematics of two-dimensional propulsive flowpath geometries. The colors delineate components of the propulsion system: inlet, isolator, combustor, and nozzle.

\section{Force and Moment Calculations}

The output of the propulsive calculations is a force per unit width and moment per unit width at the nose of each two-dimensional section. Since the propulsive geometry is two-dimensional, it is not possible to calculate a force and moment directly. If $\vec{F}_{C L}^{\prime}$ is the force per unit width of the centerline flowpath and $\vec{F}_{L}^{\prime}$ is the force per unit widh of the other flowpath geometry, the total force from the propulsion system is

$$
\vec{F}=\left(y_{C L}-y_{L}\right)\left(\vec{F}_{C L}^{\prime}+\vec{F}_{L}^{\prime}\right)
$$

where $y$ is the coordinate of the geometry in the direction perpendicular to the flow. This corresponds to trapezoidal integration in the $y$-direction. The matching equation for the moment is

$$
\vec{N}=\left(y_{C L}-y_{L}\right)\left[\vec{N}_{C L}^{\prime}+\vec{N}_{L}^{\prime}+\left(\vec{r}_{L}-\vec{r}_{C L}\right) \times \vec{F}_{L}^{\prime}\right]
$$

where $\vec{r}$ is the position at which the forces are calculated.

\section{Trim Analysis}

To demonstrate the capabilities of our reduced-order model, we used it to trim a hypersonic vehicle for steady-level Mach 8 flight at $26000 \mathrm{~m}$ altitude. The vehicle considered was the three-dimensional vehicle of Figs. 1 and 6 with the propulsive flowpath geometries from Fig. 7. The propulsive model was found to have too much noise to complete this task easily, so a curve fit model was constructed. With the curve fit model constructed we were able to trim the vehicle for this state and visualize the performance of the propulsion system. We also present the state nearest to trim obtained using the full propulsive model.

\section{A. Surrogate model and propulsion system performance}

The results from the propulsion model were highly sensitive to small changes in angle of attack and fuel-air equivalence ratio. As a result, we were not able to find a condition in which all forces and moments on the vehicle were balanced using a gradient-based minimization routine. In order to make gradient-based optimization easier, we generated a surrogate model by calculating the propulsive performance on a regularly spaced grid with 35 angles of attack and 35 fuel equivalence ratios all at the same altitude and Mach number. The results of the propulsive model for all these conditions are shown in Fig. 8.

The minimum and maximum angles of attack considered were $0^{\circ}$ and $4^{\circ}$, respectively. The minimum and maximum fuel equivalence ratios considered were 0.5 and 3.0, respectively. For conditions within these limits of angle of 
attack and equivalence ratio but not on the regular grid, the performance was calculated using cubic interpolation. The external aerodynamic model did not use a surrogate model.

The very high equivalence ratios were considered because most of the fuel does not burn even when the equivalence ratio is below 1.0. In our propulsive model, the fuel is injected from only one wall, ${ }^{10}$ which is the top wall in Fig. 7. Because of this one-sided injection and the fact that the combustor is very tall, the fuel cannot easily mix with the oxygen near the bottom of the combustor. Adding more hydrogen beyond an equivalence ratio of 1.0 simply increases diffusion. We found that an equivalence ratio of about 3.0 is needed to use all of the oxygen in the combustor. This means that either adding some of the fuel from the bottom wall of the combustor, increasing the fuel injection pressure, or using a smaller combustor would conserve fuel relative to this design.

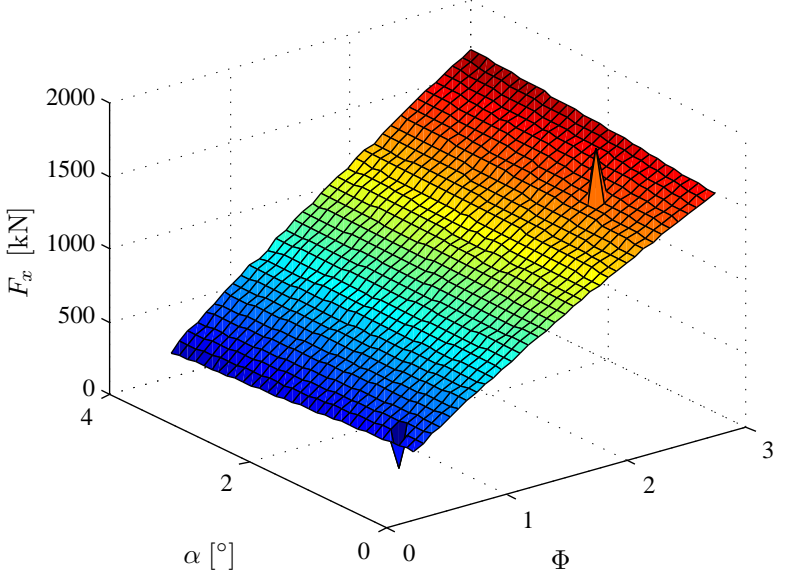

a) Installed thrust

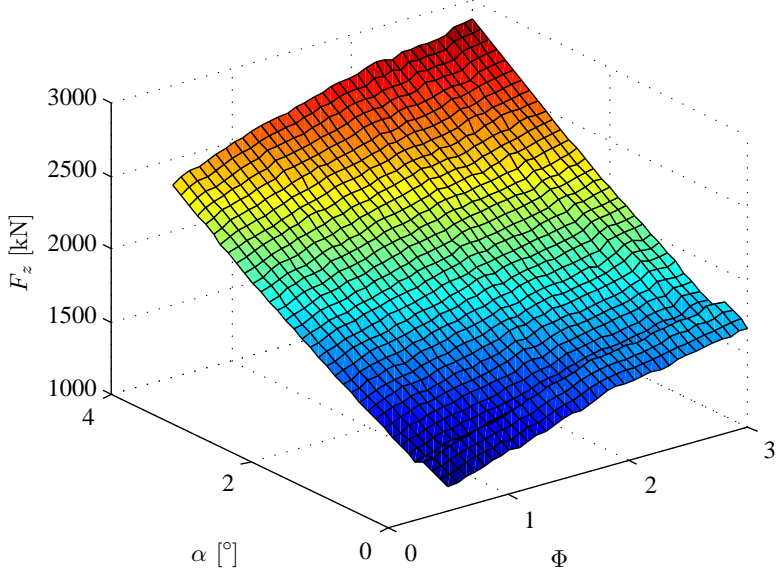

b) Inlet/nozzle total lift

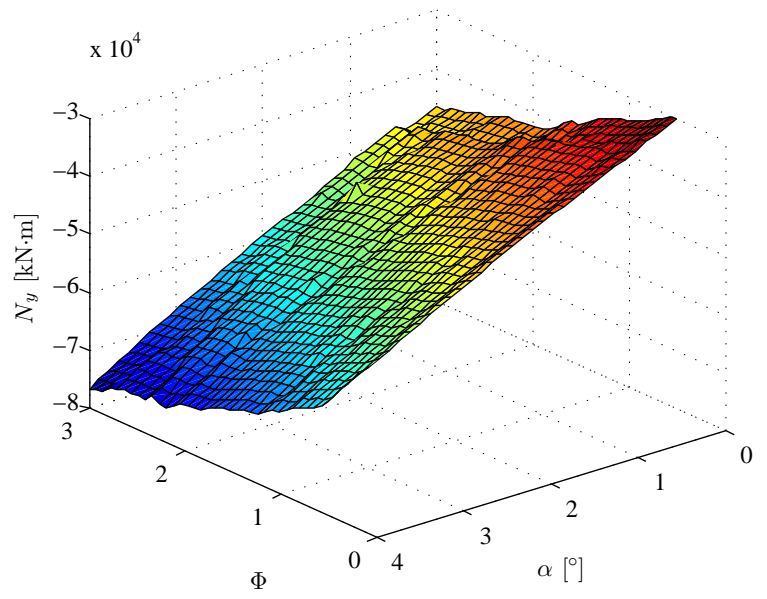

c) Pitching moment

Figure 8. Total forces and moments of propulsive components. The moment is calculated about the leading edge of the vehicle.

\section{B. Flight Dynamics Model Results}

The vehicle is trimmed for steady-level, Mach 8 flight at an altitude of $26 \mathrm{~km}$, using the two available scramjet models. This task is achieved by determining a flight condition and set of control inputs that yield zero net force and moment 
on the vehicle. Trimming can be treated as a minimization problem, where the cost function is of the form

$$
J=\left\lfloor\begin{array}{ll}
\vec{F}_{B} & \vec{N}_{B}
\end{array}\right][W]\left\{\begin{array}{l}
\vec{F}_{B} \\
\vec{N}_{B}
\end{array}\right\},
$$

where $W$ is a weighting matrix used to scale the various forces and moments. The cost function $J$ is zero at the trimmed condition and strictly positive at all other conditions. The minimization is performed numerically in MATLAB $R$ using the fmincon (constrained minimization) function. The inertia properties of the vehicle used in the following trim analysis are given in Table 1.

Table 1. Vehicle Inertia Properties Used For Trim

\begin{tabular}{ccc}
\hline \hline Symbol & Name & Value \\
\hline$m$ & Mass & $2.22 \times 10^{5} \mathrm{~kg}$ \\
$I_{x x}$ & Moment of inertia about $x$-axis & $3.42 \times 10^{6} \mathrm{~kg} \cdot \mathrm{m}^{2}$ \\
$I_{y y}$ & Moment of inertia about $y$-axis & $3.95 \times 10^{7} \mathrm{~kg} \cdot \mathrm{m}^{2}$ \\
$I_{z z}$ & Moment of inertia about $z$-axis & $3.95 \times 10^{7} \mathrm{~kg} \cdot \mathrm{m}^{2}$ \\
$I_{x y}$ & Product of inertia & $0 \mathrm{~kg} \cdot \mathrm{m}^{2}$ \\
$I_{x z}$ & Product of inertia & $0 \mathrm{~kg} \cdot \mathrm{m}^{2}$ \\
$I_{y z}$ & Product of inertia & $0 \mathrm{~kg} \cdot \mathrm{m}^{2}$ \\
\hline \hline
\end{tabular}

The trimmed values for the flight dynamics states and control variables using the surrogate propulsion model are presented in Table 2. The trimmed values using the full propulsive model are presented in Table 3. Note that $\psi, x_{E}$, and $y_{E}$ are ignored in this process because they do not contribute to the vehicle dynamics directly.

Table 2. Steady-Level Flight Trim Using Propulsion Surrogate

\begin{tabular}{ccc}
\hline \hline State & Name & Value \\
\hline$\left\|\vec{v}_{B}\right\|$ & Velocity magnitude & $2.40 \mathrm{~km} / \mathrm{s}$ \\
$-z_{E}$ & Altitude & $26 \mathrm{~km}$ \\
$\alpha$ & Angle of attack & $2.89 \mathrm{deg}$ \\
$\beta$ & Sideslip angle & $0 \mathrm{deg}$ \\
$\theta$ & Pitch Euler angle & $2.89 \mathrm{deg}$ \\
$\phi$ & Roll Euler angle & $0 \mathrm{deg}$ \\
$\dot{v}_{B, x}$ & x-component of $\dot{\vec{v}}_{B}$ & $-1.87 \times 10^{-5} \mathrm{~m} / \mathrm{s}^{2}$ \\
$\dot{v}_{B, z}$ & z-component of $\dot{\vec{v}}_{B}$ & $1.45 \times 10^{-5} \mathrm{~m} / \mathrm{s}^{2}$ \\
$\dot{\omega}_{B, y}$ & y-component of $\dot{\vec{\omega}}_{B}$ & $-1.52 \times 10^{-8} \mathrm{rad} / \mathrm{s}^{2}$ \\
$\delta_{e}$ & Elevator deflection angle & $26.9 \mathrm{deg}$ \\
$\Phi$ & Scramjet fuel-air equivalence ratio & 1.73 \\
\hline \hline
\end{tabular}

The trim state obtained using the surrogate model corresponds to a valid steady-level flight condition, because all accelerations have an acceptably small magnitude. On the other hand, the trim state obtained with the full propulsive model has a non-negligible acceleration in the $x$-direction.

In both trim cases, the elevator deflection angle is larger than expected for a hypersonic vehicle. This is necessary to correct the large nose-down pitching moment caused by the rest of the vehicle. Three possible remedies for this adverse moment are shifting the center of gravity, redesigning the nose of the vehicle, and implementing control surfaces with greater authority. The current nose of the vehicle does not have enough compression on the inlet ramp and is blunt on the top. Reversing this will provide greater compression for the engine and generate more lift near the nose. The control surfaces on the vehicle are currently limited to a pair of elevators. By either increasing the surface 
Table 3. Steady-Level Flight Trim Using Full Propulsive Model

\begin{tabular}{ccc}
\hline \hline State & Name & Value \\
\hline$\left\|\vec{v}_{B}\right\|$ & Velocity magnitude & $2.40 \mathrm{~km} / \mathrm{s}$ \\
$-z_{E}$ & Altitude & $26 \mathrm{~km}$ \\
$\alpha$ & Angle of attack & $2.86 \mathrm{deg}$ \\
$\beta$ & Sideslip angle & $0 \mathrm{deg}$ \\
$\theta$ & Pitch Euler angle & $2.86 \mathrm{deg}$ \\
$\phi$ & Roll Euler angle & $0 \mathrm{deg}$ \\
$\dot{v}_{B, x}$ & x-component of $\dot{\vec{v}}_{B}$ & $4.28 \times 10^{-1} \mathrm{~m} / \mathrm{s}^{2}$ \\
$\dot{v}_{B, z}$ & z-component of $\dot{\vec{v}}_{B}$ & $8.89 \times 10^{-5} \mathrm{~m} / \mathrm{s}^{2}$ \\
$\dot{\omega}_{B, y}$ & y-component of $\dot{\vec{\omega}}_{B}$ & $-1.59 \times 10^{-6} \mathrm{rad} / \mathrm{s}^{2}$ \\
$\delta_{e}$ & Elevator deflection angle & $27.4 \mathrm{deg}$ \\
$\Phi$ & Scramjet fuel-air equivalence ratio & 1.95 \\
\hline \hline
\end{tabular}

area of the elevators or using all-moving elevons, the control surfaces will be able to provide the necessary forces at lower deflection angles. Shifting the vehicle's center of mass a couple of meters aft would also help to counter the nose-down moment. However, the other two design modifications would still improve the vehicle's ability to maintain controlled flight for a range of conditions.

\section{Conclusion}

A reduced-order model was developed to perform rigid-body flight dynamics analysis on a hypersonic vehicle. The model can be used on a wide class of scramjet vehicles that are horizontally symmetric and have approximately two-dimensional inlets and nozzles. The model does not consider the effects of a flexible vehicle, but it provides analysis for each major component of the vehicle. For each component, the models are intended to include, at least qualitatively, all of the nonlinear behavior of a true hypersonic vehicle, including shock interactions, flameout, etc.

The only flight dynamics analysis performed in the present discussion was trimming the vehicle for steady level flight. The propulsion model was found to have unrealistic sensitivity, and a curve fit model was constructed based on the results of the propulsion model. Numerous suggestions for improvements in vehicle design were found as a result of the trim analysis. For instance a higher compression ratio in the inlet would both increase thrust and give a higher nose-up moment. Without some alterations to the design, trimming the vehicle at a selected flight condition required generating extra drag with the flaps to counteract the nose-down moment, and trimming at higher Mach numbers proved impossible. Although operation over a wide envelope of flight conditions is challenging for any aerospace vehicle, this is particularly true for hypersonic vehicles for which almost every property is nonlinear.

In addition, this framework has been extended for future models that will include aerothermoelastic effects necessary for a complete analysis of a hypersonic vehicle. This framework can also be applied as a tool in control design, control evaluation, and trajectory analysis.

\section{Acknowledgements}

This research is funded by NASA award NNX08AB32A (Don Soloway as Associate Principal Investigator) and by the Air Force Research Laboratory/Air Vehicles Directorate grant FA 8650-07-2-3744 for the Michigan/AFRL Collaborative Center in Control Sciences (Michael Bolender as technical monitor).

\section{References}

\footnotetext{
${ }^{1}$ Candler, G. V., Peterson, D. M., and Drayna, T. W., "Detached Eddy Simulation of a Generic Scramjet Inlet and Combustor," 47th AIAA Aerospace Sciences Meeting Including The New Horizons Forum and Aerospace Exposition, 2009.

${ }^{2}$ Candler, G. V. and Travis W. Drayna, T. W., "Design and Optimization of the ASET Inward-Turning Scramjet Inlet," JANAF 30th Airbreath-
} 
ing Propulsion Subcommittee Meeting, 2008.

${ }^{3}$ Higgins, K. and Schmidt, S., "Simulation of a Sonic Jet Injected into a Supersonic Cross-Flow," 16th Australasian Fluid Mechanics Conference, 2007.

${ }^{4}$ Oppenheimer, M. W., Skujins, T., Bolender, M. A., and Doman, D. B., "A Flexible Hypersonic Vehicle Model Developed with Piston Theory," Proceedings of the 2007 Atmospheric Flight Mechanics Conference and Exhibit, AIAA Paper No. 2007-6396, August 2007.

${ }^{5}$ Bolender, M. A. and Doman, D. B., "Nonlinear Longitudinal Dynamical Model of an Air-Breathing Hypersonic Vehicle," Journal of Spacecraft and Rockets, Vol. 44, No. 2, 2007, pp. 374-387.

${ }^{6}$ Chavez, F. R. and Schmidt, D. K., "Analytical Aeropropulsive/Aeroelastic Hypersonic-Vehicle Model with Dynamic Analysis,” Journal of Guidance, Control, and Dynamics, Vol. 17, No. 6, 1994, pp. 1308-1319.

${ }^{7}$ Mirmirani, M., Wu, C., Clark, A., Choi, S., and Colgren, R., "Modeling for Control of a Generic Airbreathing Hypersonic Vehicle," Proceedings of the 2005 Guidance, Navigation and Control Conference, AIAA Paper No. 2005-6256, August 2005.

${ }^{8}$ O'Brien, T. F., Starkey, R. P., and Lewis, M. J., "Quasi-One-Dimensional High-Speed Engine Model with Finite-Rate Chemistry," Journal of Propulsion and Power, Vol. 17, No. 6, 2001, pp. 1366-1374.

${ }^{9}$ Torrez, S. M., Scholten, N. A., Micka, D. J., Driscoll, J. F., Bolender, M. A., Doman, D. B., and Oppenheimer, M. W., "A Scramjet Engine Model Including Effects of Precombustion Shocks and Dissociation," 44th AIAA/ASME/SAE/ASEE Joint Propulsion Conference \& Exhibit, 2008, AIAA Paper No. 2008-4619.

${ }^{10}$ Torrez, S. M., Driscoll, J. F., Dalle, D. J., and Micka, D. J., "Scramjet Engine Model MASIV: Role of Mixing, Chemistry and Wave Interaction," 45th AIAA/ASME/SAE/ASEE Joint Propulsion Conference and Exhibit, 2009, AIAA Paper No. $2009-4939$.

${ }^{11}$ Frendreis, S. G. V., Skujins, T., and Cesnik, C. E. S., "Six-Degree-of-Freedom Simulation of Hypersonic Vehicles," AIAA Atmospheric Flight Mechanics Conference, 2009, AIAA Paper 2009-5601.

${ }^{12}$ Dalle, D. J., Fotia, M. L., and Driscoll, J. F., "Reduced-Order Modeling of Two-Dimensional Supersonic Flows with Applications to Scramjet Inlets," Journal of Propulsion and Power, Vol. 26, No. 3, 2010, pp. 545-555.

${ }^{13}$ Dalle, D. J., Torrez, S. M., and Driscoll, J. F., "Reduced-Order Modeling of Reacting Supersonic Flows in Scramjet Nozzles," 46th AIAA/ASME/SAE/ASEE Joint Propulsion Conference and Exhibit, 2010.

${ }^{14}$ Vogel, J. M., Kelkar, A. G., Inger, G., Whitmer, C., Sidlinger, A., and Rodriguez, A., "Control-Relevant Modeling of Hypersonic Vehicles," 2009 American Control Conference, 2009.

${ }^{15}$ Torrez, S. M., Driscoll, J. F., Dalle, D. J., Bolender, M. A., and Doman, D. B., "Hypersonic Vehicle Thrust Sensitivity to Angle of Attack and Mach Number," AIAA Atmospheric Flight Mechanics Conference, 2009, AIAA Paper No. 2009-6152.

${ }^{16}$ Torrez, S. M., Driscoll, J. F., Dalle, D. J., and Fotia, M. L., "Preliminary Design Methodology for Hypersonic Engine Flowpaths," 16th AIAA/DLR/DGLR International Space Planes and Hypersonic Systems and Technologies Conference, 2009. 